\title{
Sweet's Syndrome Associated with Acute-Onset Encephalitis
}

\author{
In-Uk Song Joong-Seok Kim Yeong-In Kim Kwang-Soo Lee \\ Department of Neurology, College of Medicine, The Catholic University of Korea, Seoul, South Korea
}

\section{Dear Sir,}

Sweet's syndrome is also known as acute febrile neutrophilic dermatosis, and it is a distinct syndrome originally described by Sweet in 1966 [1]. This syndrome is an unusual disease that is characterized by malaise, fever, leukocytosis and distinctive skin lesions [2]. The lesions heal without scarring, and they are typically tender, dull-red edematous plaques that usually occur on the face, neck and the upper part of the trunk and limbs [3]. Skin biopsies reveal a dense dermal infiltration of neutrophils with the spared epidermis, and there are not any histological signs of vasculitis [2, 4]. Increased peripheral blood neutrophils, C-reactive protein and an increased erythrocyte sedimentation rate (ESR) are common findings [3]. Arthralgia, arthritis, conjunctivitis and iridocyclitis are also common [5]. While various malignant neoplasms and inflammatory or autoimmune disorders such as rheumatoid arthritis, ulcerative colitis, Sjogren disease and Behcet's disease have been reported to be associated with Sweet's syndrome, there are only rare reports of central nervous system (CNS) involvement $[5,6]$. The pathogenesis of this syndrome remains unknown, although it is believed to represent a hypersensitivity reaction to an infection or malignancy [7]. We described here a patient with Sweet's syndrome that was associated with acute-onset encephalitis.

Case Report

A 59-year-old man was seen in the Department of Dermatology 3 years ago; he presented with fever and tender, dell-red edematous plaques on the face, neck, upper part of the trunk and right forearm. He was diagnosed as having Sweet's syndrome because of his clinical findings and a biopsy that revealed leukocytosis and a dense dermal infiltrate composed of mature neutrophils with nuclear fragmentations in an edematous plaque on his right forearm (fig. 1). He had been treated with oral corticosteroids (prednisolone 10-20 mg/day). However, he had stopped corticosteroid therapy on his own about 10 months ago.

He was admitted to our hospital for an acute exacerbation of disease, and he suddenly showed mental changes and high fever that was associated with malaise and erythematous skin plaques on the right forearm, face and neck (fig. 2). On admission day, the vital signs showed a body temperature of $39.8^{\circ} \mathrm{C}$, a blood pressure of $130 / 80 \mathrm{~mm} \mathrm{Hg}$, a pulse rate of $99 / \mathrm{min}$ and a respiratory rate of $30 / \mathrm{min}$. The physical examination revealed multiple tender, dull-red erythematous plaques on the left zygomatic area and periocular area, the upper part of the trunk and the right forearm. No genital ulcer, folliculitis-like eruptions, erythema nodosum-like lesions or ophthalmic involvement were noted. On neurologic examination, he revealed a stuporous mental state, hyperactive deep tendon reflexes and neck stiffness. The CSF studies revealed clear color fluid, normal pressure, $61 / \mathrm{mm}^{3}$ white blood cells, $3 / \mathrm{mm}^{3}$ red blood cells, a protein level of $39.5 \mathrm{mg} / \mathrm{dl}$, a normal glucose level. Oligo- clonal band and antibodies against herpes simplex virus, Japanese encephalitis and cytomegalovirus were not present. The complete blood cell count showed nonspecific findings except for leukocytosis with $85.5 \%$ neutrophils. The ESR was 72 $\mathrm{mm} / \mathrm{h}$. The other laboratory findings, including the serum chemistry, liver function testing, cholesterol, the prothrombin and partial thromboplastin time and urinalysis, were all within normal limits. In addition, alpha-fetoprotein, carcinoembryonic antigen (CEA), rheumatoid factor, perinuclear antineutrophil cytoplasmic antibodies (p-ANCA), anticardiolipin antibodies, antinuclear antibodies (ANA) and the bone marrow biopsy were also normal. A culture of the CSF and blood was negative for bacteria, tuberculosis and fungi. Human leukocyte antigen (HLA) typing showed B-54 and Cw1. On the brain MRI studies, high signal intensities on the bilateral basal ganglia were noted on the T2WI and FLAIR images (fig. 3). The MR angiography showed a normal appearance. The electroencephalogram studies revealed diffuse mixed slowing waves (mainly, delta wave) on both hemispheres (fig. 4). Considering the above results, we excluded infectious disease, cerebrovascular disease, Behcet disease and demyelinating disease from our differential diagnosis. The concomitant development of neurologic symptoms with the skin lesions of Sweet's syndrome suggested a diagnosis of Sweet's syndrome with neurologic manifestations. Thus, we started corticosteroid

\section{KARGER}

Fax +41613061234 E-Mail karger@karger.ch www.karger.com
(C) 2007 S. Karger AG, Basel 0014-3022/07/0573-0182\$23.50/0

Accessible online at: www.karger.com/ene
Dr. Kwang-Soo Lee

Department of Neurology, Kangnam St. Mary's Hospital

505, Banpo-dong, Seocho-gu

Seoul 137-701 (South Korea)

Tel. +82 2590 2091, Fax +82 2599 9686, E-Mail ks1007@catholic.ac.kr 


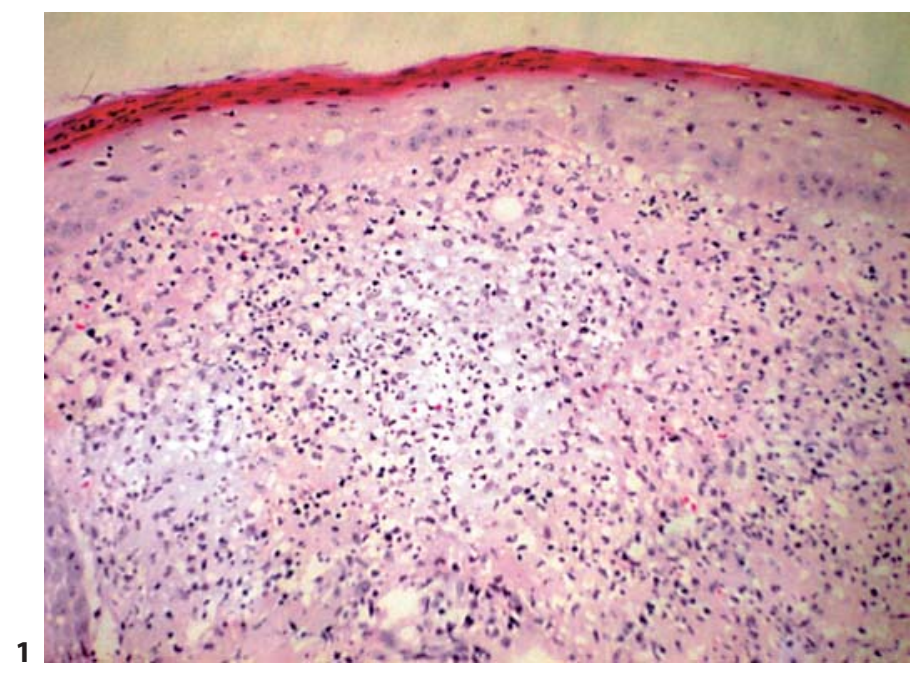

Fig. 1. The dense neutrophilic infiltrate on the upper one third is consistent with acute frebrile neurophilic dermatosis (right arm). HE. $\times 100$.

Fig. 2. Clinical photograph of the patient showing the dull-red, edematous plaque on the face.

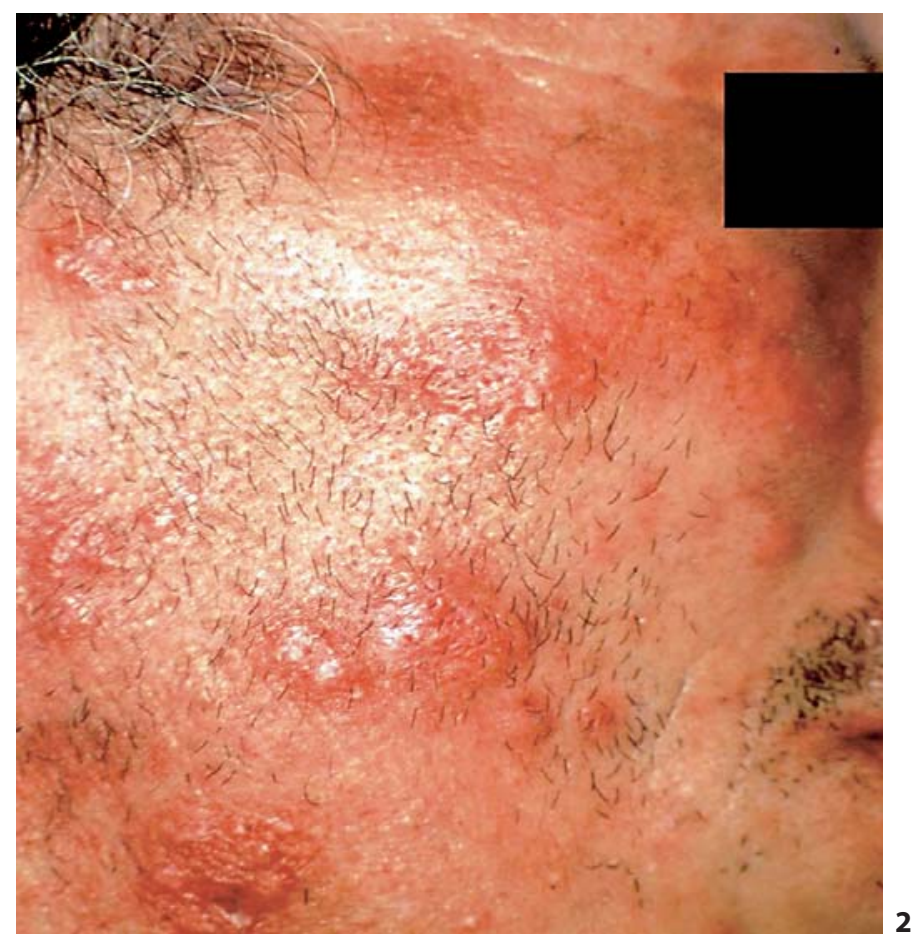

Fig. 3. Brain MRI (FLAIR) shows bilateral hyperintensities in the both basal ganglia with high signal foci in both caudate nucleus heads.

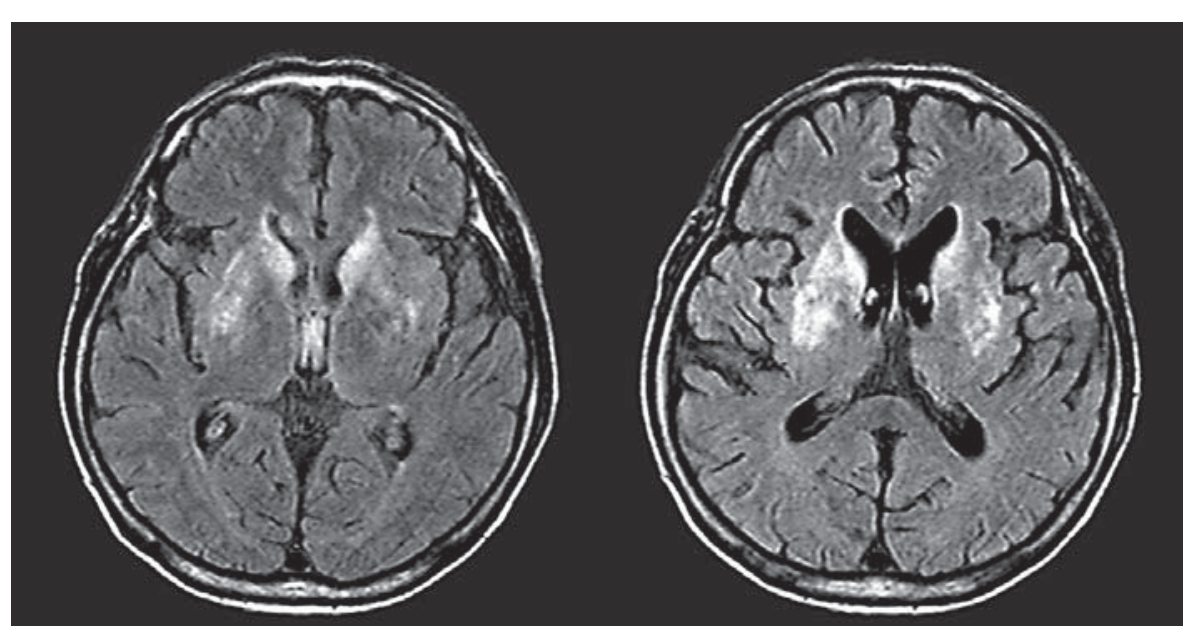

Discussion

There have been only a few case reports of CNS involvement in Sweet's syndrome [3]. Chiba et al. [6] first reported a patient with Sweet's syndrome who presented with neuropsychiatric symptoms that included disorientation, convulsion, myoclonus and depression. The CT scan revealed only brain atrophy, but there was no pleocytosis in CSF. Druschky et al. [8] have reported a patient with meningitis and hemiparesis, but the MRI failed to show any lesions. Hisanaga et al. [5] in 1999 reported on a patient who had Sweet's syndrome with encephalitis in the deep brain structure, as determined by MRI. These lesions consisted of high signal intensities on the T2WIs in the thalamus, putamen, caudate nucleus, midbrain and cerebral white matter [5]. The authors 
Fig. 4. Waking EEG of the patient shows diffuse mixed slowing waves (mainly, delta waves) on both hemispheres.

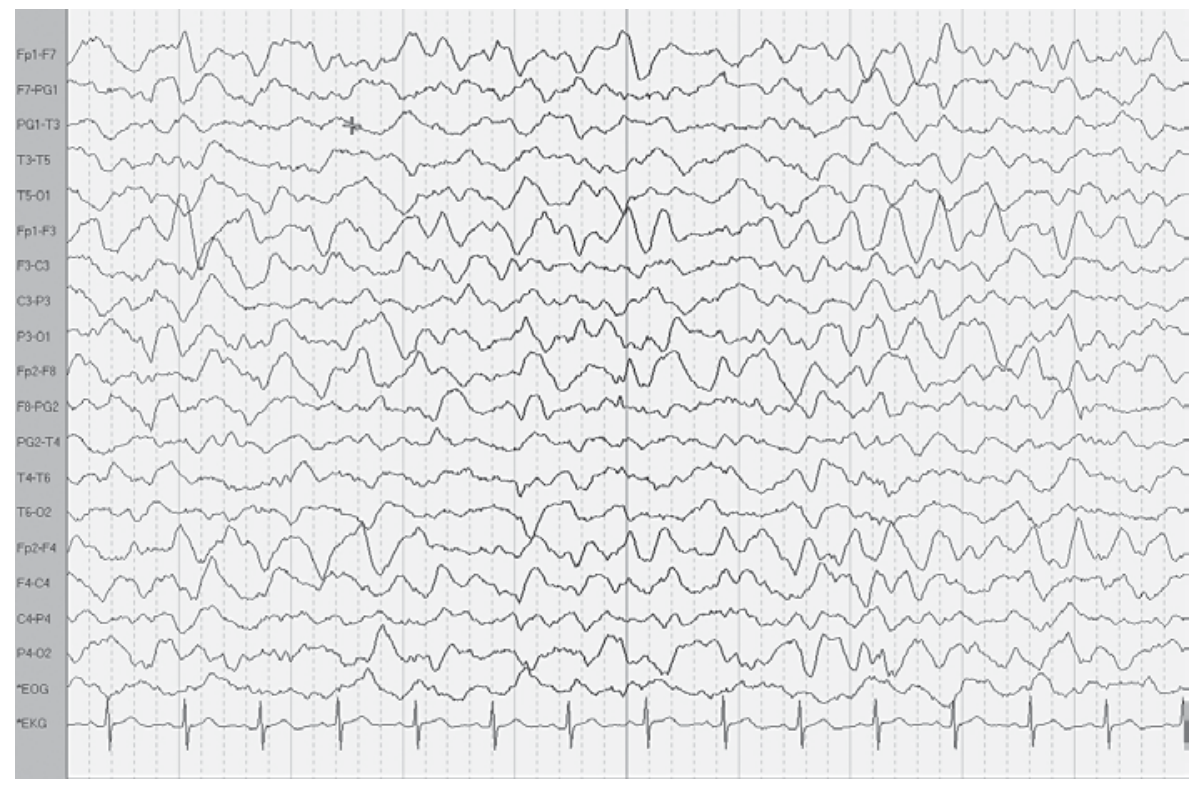

drome. Granulocyte colony-stimulating factor therapy for treating the granulopenia is known to induce Sweet's syndrome. Further studies are needed to investigate the association of the cytokine system with Sweet's syndrome, especially in reference to the CNS involvement.

Sweet's syndrome itself, regardless of the involvement of the CNS, may be a benign disorder unless another serious disease such as malignancy is associated with it [6]. Moreover, the CNS involvement in Sweet's syndrome is usually transient, but this may be recurrence in some cases [5]. The CNS involvement may become more recognizable if the CSF and/or the brain image on MRI are extensively examined. Awareness of the neurological complications in Sweet's syndrome is important because it may help to avoid unnecessary empiric therapy for meningoencephalitis and so lead to successful treatment with systemic corticosteroids. Therefore, like in this present case, we emphasize that investigating the neurologic manifestations may be required, although CNS involvement in Sweet's syndrome has rarely been reported.

\section{References}

1 Sweet RD: An acute febrile neutrophilic dermatosis. Br J Dermatol 1964;74:349-356.

2 Nobeyama Y, Kamide R: Sweet's syndrome with neurologic manifestation: case report and literature review. Int J Dermatol 2003; 42:438-443.
3 Hisanaga K, Iwasaki Y, Itoyama Y, et al: Neuro-Sweet disease: clinical manifestations and criteria for diagnosis. Neurology 2005; 64:1756-1761.

4 Going JJ, Going SM, Myskow MW, Beverdge GW: Sweet's syndrome: histological and immunohistochemical study of 15 cases. J Clin Pathol 1987;40:175-179.

5 Hisanaga K, Hosokawa M, Sato N, Mochizuki H, Itoyama Y, Iwasaki Y: 'Neuro-Sweet disease' benign recurrent encephalitis with neurotrophilic dermatosis. Arch Neurol 1999;58:1010-1013.

6 Kazuyuki N, Yasuyuki O, Jiro F, Kenji F, Keigo G, Hiroko S, et al: Sweet's syndrome associated with encephalitis. J Neurol Sci 2001; 188:95-97.

7 Kim YK, Bang DS: Sweet syndrome associated with bacterial meningitis. Ann Dermatol 1996;8:766-769.

8 Druschky A, von den Driesch P, Anders M, Claus D, Neundorfer B: Sweet's syndrome (acute febrile neutrophilic dermatosis) affecting the central nervous system. J Neurol 1996;243:556-557.

9 Fitzgerald RL, McBurney EI, Nesbitt LT Jr: Sweet's syndrome. Int J Dermatol 1996;35: 9-15.

10 Mizoguchi M, Matsuki K, Mochizudi M, Watanabe R, Orawa K, Harada S et al: $\mathrm{Hu}-$ man leukocyte antigen in Sweet's syndrome and its relationship to Behcet's disease. Arch Dermatol 1988;124:1069-1073. hypersensitivity reaction to a bacterial, viral or tumor antigen [7]. Cytokines, including granulocyte colony-stimulating factor, IL-6 and ANCA, have been implicated in the pathogenesis of Sweet's syn- 